Pacific Journal of Mathematics

THE ENDOMORPHISMS OF A DIRICHLET ALGEBRA 


\title{
THE ENDOMORPHISMS OF A DIRICHLET ALGEBRA
}

\author{
BRUCE LUND
}

\begin{abstract}
Let $A(A)$ be the disc algebra. A Dirichlet subalgebra of $A(\Delta)$ is obtained by forming $A(p)=A(\Delta) \cap\{f \circ p: f \in A(\Delta)\}$ where $p\left(e^{i t}\right)$ in a singular homeomorphism of the unit circle. In this paper the endomorphisms of $A(p)$ are described and for a restricted class of these endomorphisms the spectra is computed.
\end{abstract}

1. Introduction. The disc algebra $A(\Delta)$ is the uniform algebra of functions analytic on the open unit disc $\Delta=\{z:|z|<1\}$ and continuous on the closure $\bar{J}$. In [3] Browder and Wermer gave a method for the construction of algebras of $A(\Delta)$ which are Dirichlet algebras on the unit circle $\Gamma=\{z:|z|=1\}$. Their method goes as follows: Let $p\left(e^{i t}\right)$ be a homeomorphism of $\Gamma$ where $p^{\prime}\left(e^{i t}\right)=0$ for almost all $t$. Such homeomorphisms will be called singular. Let $A(\Delta)_{p}=$ $\{f \circ p: f \in A(\Delta)\}$ and set $A(p)=A(\Delta) \cap A(\Delta)_{p}$. Then $A(p)$ is a Dirichlet algebra on $\Gamma$. Properties of $A(p)$ are given in [1], [2], [8].

In $\S 2$ we show that two singular homeomorphisms $p_{1}$ and $p_{2}$ may lead to nonisomorphic algebras $A\left(p_{1}\right)$ and $A\left(p_{2}\right)$. Nonetheless, some general characteristics of the space of endomorphisms of an algebra $A(p)$ are given in $\S 3$. In $\S 4$ the techniques of Kamowitz [4], [5] are applied to describe the spectra of certain of the endomorphisms of $A(p)$.

We collect some information about the algebra $A(p)$ and refer the reader to [7], Section 8.2 for further discussion. To describe the maximal ideal space $M_{A(p)}$ of $A(p)$, we first make the convention that if $f \in A(p)$, then $\widetilde{f \circ p}$ will stand for the analytic extension of $f \circ p\left(e^{i t}\right)$ to $\Delta$. Also, we let $f(y)$ denote the value of $f \in A(p)$ evaluated at the maximal ideal $y$. Then $M_{A(p)}$ consists of $\Gamma$ and two parts lying off $\Gamma$. One of these parts is identified with $\Delta$ and the other, to be denoted by $\Delta_{p}$, consists of points of the form $\Psi(z)$ where

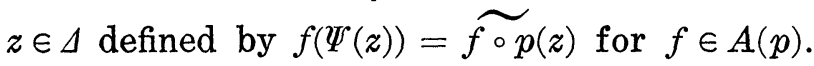

When the maximal ideal space is given the Gelfand topology, we have $\bar{\Delta} \backslash \Delta=\Gamma$ and $\bar{\Delta}_{p} \backslash \Delta_{p}=\Gamma$. The Gelfand topology coincides with the usual topology on $\bar{\Delta}$ and the map $\Psi: \Delta \rightarrow \Delta_{p}$ is a homeomorphism. Moreover, $\Psi$ extends to $\Gamma$ to map $\bar{\Delta}$ homeomorphically onto $\overline{\Delta_{p}}$ by setting $\Psi\left(e^{i t}\right)=p\left(e^{i t}\right)$.

Given $z_{0} \in \Delta$ we claim there are functions $f \in A(p)$ such that $f^{\prime}\left(z_{0}\right) \neq 0$. Likewise, there are functions $f \in A(p)$ such that 
$\widetilde{f \circ p^{\prime}}\left(z_{0}\right) \neq 0$. We verify this claim for $z_{0}=0$ as an illustration of the general case. Suppose instead that every $f \in A(p)$ satisfied $f^{\prime}(0)=0$. Then $\mu=[(1+\cos (t)) / 2 \pi] d t$ is a representing measure for the origin which is supported on $\Gamma$. But normalized Lebesque measure on $\Gamma$ also is a representing for the origin contradicting the uniqueness of representing measures for a Dirichlet algebra.

As a result of these observations we conclude:

(1) Given $z_{0} \in \Delta$ and a positive integer $k$ there is $f \in A(p)$ such that $f(z)=\left(z-z_{0}\right)^{k} g(z)$ where $g\left(z_{0}\right) \neq 0$. Similarly, there is $f \in A(p)$ such that $\widetilde{f \circ p}(z)=\left(z-z_{0}\right)^{k} h(z)$ where $h\left(z_{0}\right) \neq 0$.

(2) If $g: \bar{\Delta} \rightarrow \bar{\Delta}$ is continuous and $f \circ g \in A(\Delta)$ for all $f \in A(p)$, then $g \in A(\Delta)$. Similarly, if $f \circ \Psi \circ g \in A(\Delta)$ for all $f \in A(p)$, then $g \in A(\Delta)$.

2. Isomorphisms. Let $A(p)$ and $A(q)$ be two Dirichlet algebras as described in $\S 1$. If $T$ is a homomorphism (linear and multiplicative) from $A(p)$ to $A(q)$, then $T$ induces a continuous mapping $\Phi: M_{A(q)} \rightarrow M_{A(p)}$ defined as follows: If $y \in M_{A(q)}$, then $\Phi(y)$ is the maximal ideal which satisfies $f(\Phi(y))=T(f)(y)$ for all $f \in A(p)$. We call $\Phi$ the mapping induced by $T$. The mapping $\Phi$ has the following properties:

(a) $\Phi^{-1}(\Gamma) \subset \Gamma$.

(b) Either $\Phi(\Delta) \subset \Delta$ or $\Phi(\Delta) \subset \Delta_{p}$.

(c) Either $\Phi\left(\Delta_{q}\right) \subset \Delta$ or $\Phi\left(\Delta_{q}\right) \subset \Delta_{p}$.

To establish (a) we suppose there exists $x \in \Gamma$ and $y \in \Phi^{-1}(x)$ where $y \notin \Gamma$. Each point of $\Gamma$ is a peak point for $A(q)$. Let $f \in A(q)$ peak at $x$. Then $T(f)(y)=f \circ \Phi(y)=f(x)$ and $T(f)$ peaks at $y$. However, this contradicts the maximum principle when $y \notin \Gamma$. Properties (b) and (c) follow from (a) and the fact that $\Delta, \Delta_{p}$, and $\Delta_{q}$ are open and connected.

In order to state the following theorem we let $\Psi: \bar{\Delta} \rightarrow \bar{\Delta}_{p}$ be

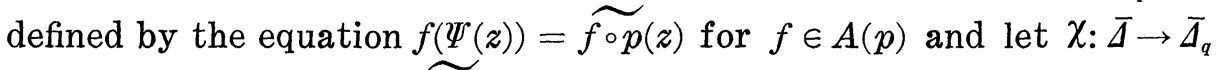

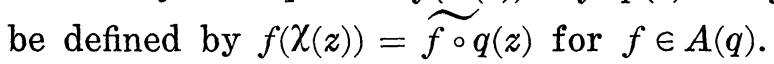

THEOREM 1. Let $T: A(p) \rightarrow A(q)$ be an isomorphism and let $\Phi: M_{A(q)} \rightarrow M_{A(p)}$ be the induced map. Then $\Phi$ is a homeomorphism with $\Phi(\Gamma)=\Gamma$ and either (i) $\Phi(\Delta)=\Delta, \Phi\left(\Delta_{q}\right)=\Delta_{q}$ and $\Phi \in A(\Delta)$, $\Psi^{-1} \circ \Phi \circ \chi \in A(\Delta)$. (Hence, $\Phi$ and $\Psi^{-1} \circ \Phi \circ \chi$ are conformal maps of $\Delta$ onto itself.) $O r$ (ii) $\Phi(\Delta)=\Delta_{p}, \Phi\left(\Delta_{q}\right)=\Delta$ and $\Psi^{-1} \circ \Phi \in A(\Delta), \Phi \circ \chi \in A(\Delta)$. (Hence, $\Psi^{-1} \circ \Phi$ and $\Phi \circ \chi$ are conformal maps of $\Delta$ onto itself.)

Proof. It is easy to check that $\Phi$ is a homeomorphism with $\Phi(\Gamma)=\Gamma$. Either $\Phi(\Delta) \subset \Delta$ or $\Phi(\Delta) \subset \Delta_{p}$. Suppose $\Phi(\Delta) \subset \Delta . \quad$ Then 
$\Phi\left(\Delta_{q}\right) \subset \Delta_{p}$ or else $\Delta_{p}$ would be omitted from the range of $\Phi$ altogether. Again, since $\Phi$ is onto we conclude $\Phi(\Delta)=\Delta$ and $\Phi\left(\Delta_{q}\right)=\Delta_{p}$. Using Property 2) listed in $\S 1$ we conclude that $\Phi$ and $\Psi^{-1} \circ \Phi \circ \chi \in A(\Delta)$. Consideration of the case $\Phi(\Delta) \subset \Delta_{p}$ leads to alternative (ii). This completes the proof.

CoRollary. If $p\left(e^{2 t}\right)$ and $q\left(e^{i t}\right)$ give different orientations to $\Gamma$, then $A(p)$ and $A(q)$ are not isomorphic.

Proof. Assume alternative (i) of the theorem. Then $\Psi^{-1} \circ \Phi \circ \chi$ is analytic on $\bar{\Delta}$ and maps $\Gamma$ homeomorphically onto itself. However, the orientation of $\Gamma$ is reversed by this map which gives us a contradiction. A similar argument applies to alternative (ii).

The next example shows that $A(p)$ and $A(q)$ need not be isomorphic when $p$ and $q$ give the same orientation to $\Gamma$.

EXAMPLE 1. In [10] a strictly increasing, continuous, singular function $h(x)$ is constructed which maps the interval $[0,1]$ onto itself. Furthermore, $h(x)$ has the property that if $h^{\prime}\left(x_{0}\right)$ exists for some $x_{0} \in(0,1]$, then $h^{\prime}\left(x_{0}\right)=0$. Also, if $\rho=3$ where $\rho$ is a constant appearing in [10], then it follows that $h^{\prime}(0)=0$. (Derivatives at 0 and 1 are one-sided.) For such an $h(x)$ we define $p\left(e^{i t}\right)=e^{i 2 \pi h(t)}$.

Next, we indicate how to construct a strictly increasing, continuous, singular function $k(x)$ taking $[0,1]$ onto itself which satisfies $k^{\prime}(0)=1$ and $k^{\prime}(1)=1$. To obtain $k^{\prime}(0)=1$ we set $\alpha(x)=e^{x}-1$ and $\beta(x)=\alpha^{-1}(x)$ and construct $k(x)$ to lie between $\alpha(x)$ and $\beta(x)$. A similar approach applies to obtain $k^{\prime}(1)=1$. Now define $q\left(e^{i t}\right)=$ $e^{i 2 \pi k(t)}$.

Suppose $T$ is an isomorphism of $A(p)$ to $A(q)$ with induced map $\Phi$. Assume alternative (i) of Theorem 1 holds. Let $g$ be given by $g=p^{-1} \circ \Phi \circ q$. Then $g$ and $\Phi$ are differentiable on $\Gamma$ with nonvanishing derivative. Consider $\Phi \circ q=p \circ g$. At $t=0, \Phi \circ q$ is differentiable with nonzero derivative. This implies that $p$ is differentiable at $g(0)$ with nonzero derivative which is a contradiction. A similar argument will eliminate the possibility of alternative (ii). Hence, $A(p)$ and $A(q)$ are not isomorphic.

REMARK. It is easy to construct algebras $A(p)$ and $A(q)$ which are isomorphic. For example, given $p$ we let $q=g^{-1} \circ p \circ g$ where $g$ is a conformal map of the disc onto itself. Then $A(p)$ and $A(q)$ are isomorphic by the map $T: A(p) \rightarrow A(q)$ where $T(f)=f \circ g$.

3. Endomorphisms of $A(p)$. Let $T$ be endomorphism of $A(p)$ with induced map $\Phi$. There are four cases to be considered. 
Case (i). $\Phi(\Delta) \subset \Delta$ and $\Phi\left(\Delta_{p}\right) \subset \Delta$.

In this case $T(f)(z)=f(\Phi(z)) \in A(\Delta)$ for all $f \in A(p)$ and thus $\Phi \in A(\Delta)$. Likewise, $\quad T(f) \circ \Psi(z)=f(\Phi \circ \Psi(z)) \in A(\Delta)$ for all $f \in A(p)$ and thus $\Phi \circ \Psi \in A(\Delta)$. We conclude that $\Phi \in A(p)$ and $\|\Phi\| \leqq 1$. Such endomorphisms exist since to each $\Phi \in A(p)$ where $\|\Phi\| \leqq 1$ we can associate an endomorphism $T_{\Phi}$ where $T_{\Phi}(f)=f \circ \Phi$.

Case (ii). $\Phi(\Delta) \subset \Delta_{p}$ and $\Phi\left(\Delta_{p}\right) \subset \Delta_{p}$.

In this case $T(f)(z)=f(\Phi(z))=f \circ \Psi\left(\Psi^{-1} \circ \Phi(z)\right) \in A(\Delta)$ for all $f \in A(p)$ and thus $\Psi^{-1} \circ \Phi \in A(\Delta)$. A similar argument shows $\Psi^{-1} \circ \Phi \circ \Psi \in A(\Delta)$. We conclude that $\Psi^{-1} \circ \Phi \in A(p)$ and $\left\|\Psi^{-1} \circ \Phi\right\| \leqq 1$. Such endomorphisms exist since to each $g \in A(p)$ where $\|g\| \leqq 1$ we can associate an endomorphism $T^{g}$ where $T^{g}(f)=f \circ \Psi \circ g$. If $\Phi$ is the map induced by $T^{g}$, then $\Phi(z)=\Psi(g(z))$ and $\Phi(\Psi(z))=\Psi \circ g(\Psi(z))$.

Case (iii). $\Phi(\Delta) \subset \Delta$ and $\Phi\left(\Delta_{p}\right) \subset \Delta_{p}$.

In this case $\Phi(\Gamma)=\Gamma$. By arguments similar to those in cases (i) and (ii) we have $\Phi$ and $\Psi^{-1} \circ \Phi \circ \Psi$ in $A(\Delta)$. Hence, $\Phi$ and $\Psi^{-1} \circ \Phi \circ \Psi$ are finite Blaschke products and $\Phi(\Delta)=\Delta, \Phi\left(\Delta_{p}\right)=\Delta_{p}$.

Examples of such endomorphisms may be given for particular $p\left(e^{i t}\right)$. Example 2 was suggested by W. Knight.

EXAMPLE 2. Let $\alpha(x)$ be a strictly increasing, continuous, singular function mapping $[0, \pi]$ onto $[\pi, 2 \pi]$. Extend $\alpha$ to $(\pi, 2 \pi]$ by defining $\alpha(x)=\alpha(x-\pi)-\pi$ and extend $\alpha$ to $R$ by periodicity. Set $p\left(e^{i t}\right)=e^{i \alpha(t)}$ and notice that $p$ is a singular homeomorphism of $\Gamma$. Let $\Phi\left(e^{i t}\right)=-e^{i t}$. It can be readily verified that $p \circ \Phi\left(e^{i t}\right)=$ $\Phi \circ p\left(e^{i t}\right)$. Consider $A(p)$ and let $T(f)=f \circ \Phi$. Using the equation $p \circ \Phi=\Phi \circ p$ we see that $T$ is an automorphism of $A(p)$ which falls into case (iii).

Case (iv). $\Phi(\Delta) \subset \Delta_{p}$ and $\Phi\left(\Delta_{p}\right) \subset \Delta$.

By arguments similar to those in the previously considered cases we see that $\Phi(\Gamma)=\Gamma, \Phi(\Delta)=\Delta_{p}, \Phi\left(\Delta_{p}\right)=\Delta$ and that $\Psi^{-1} \circ \Phi$ and $\Phi \circ \Psi$ belong to $A(\Delta)$.

Examples of such endomorphisms may be given for particular $p\left(e^{i t}\right)$.

EXAMPLE 3. Let $p\left(e^{\imath t}\right)$ be a singular homeomorphism of $\Gamma$ which satisfies $p \circ p\left(e^{i t}\right)=e^{i t}$. See [7] p. 207 for a construction. Set $T(f)=f \circ p$ for $f \in A(p)$. Then $T$ is an automorphism of $A(p)$ which falls into case (iv).

The next result gives a property of certain automorphisms of $A(p)$. We adopt the following notation. If $T$ is an automorphism, 
then $T^{n}=T \circ \ldots \circ T$ ( $n$ times) and $I$ will denote the identity automorphism.

THEOREM 2. Let $T$ be an automorphism of $A(p)$ such that the induced map $\Phi$ satisfies $\Phi(\Delta) \subset \Delta$ and $\Phi\left(\Delta_{p}\right) \subset \Delta_{p}$. If either $\Phi$ or $\Psi^{\sim} \circ \Phi \circ \Psi$ has a fixed point in $\Delta$, then $T$ is a nilpotent element in the group of automorphisms of $A(p)$.

Proof. We assume first that $\Phi(0)=0$. Then $\Phi\left(e^{i t}\right)=c e^{i t}$ where $|c|=1$. Assume there is no positive integer $n$ for which $c^{n}=1$. Then $\left\{c, c^{2}, c^{3}, \cdots\right\}$ is dense in $\Gamma$. Let $\alpha_{0} \in \Gamma$ be given. We will show that the map $U_{0}$ on $A(p)$ defined by $U_{0}(f)\left(e^{i t}\right)=f\left(\alpha_{0} e^{i t}\right)$ defines an automorphism of $A(p)$. Let $\alpha_{n}$ be a sequences taken from $\left\{c, c^{2}, c^{3}, \cdots\right\}$ which converses to $\alpha_{0}$. To see that $U_{0}$ maps $A(p)$ to $A(p)$, we let $f \in A(p)$ and note that $f\left(\alpha_{n} e^{i t}\right) \in A(p)$ for each $n$ and that $f\left(\alpha_{n} e^{i t}\right)$ converges to $f\left(\alpha_{0} e^{i t}\right)$ uniformly on $\Gamma$. It is evident that $U_{0}$ is an endomorphism and it is not difficult to verify that it is actually an automorphism with induced map satisfying case (iii). In particular, $p^{-1}\left(\alpha_{0} p\left(e^{i t}\right)\right)$ has nonvanishing derivative since this function gives the boundary values of a conformal map of the disc.

Let $e^{i t_{0}}$ be a point where the derivative of $p\left(e^{i t}\right)$ vanishes. Then select $\alpha_{0} \in \Gamma$ so that $\alpha_{0} p\left(e^{i t_{0}}\right)$ is a point where the derivative of $p^{-1}\left(e^{i t}\right)$ vanishes. In this event the derivative of $p^{-1}\left(\alpha_{0} p\left(e^{i t}\right)\right)$ vanishes at $e^{i t_{0}}$ which gives a contradiction. Consequently, there must be an integer $n>0$ for which $c^{n}=1$. But then $T^{n}=I$.

If $\Phi\left(z_{0}\right)=z_{0} \in \Delta$ and $z_{0} \neq 0$, then let $g(z)$ be a conformal map of $\Delta$ taking 0 to $z_{0}$. Set $q=g^{-1} \circ p \circ g$ and $\Theta=g^{-1} \circ \Phi \circ g$. An automorphism of $A(q)$ is given by $T^{\prime}$ where $T^{\prime}(f)=f \circ \Theta$. Since $\Theta(0)=0$, the previous results apply to show $T^{\prime}$ nilpotent. It follows that $T$ is nilpotent.

Now assume $\Psi^{-1} \circ \Phi \circ \Psi\left(z_{0}\right)=z_{0} \in \Delta$. An automorphism of $A\left(p^{-1}\right)$ is defined by $T_{1}$ where $T_{1}(f)=f\left(\Psi^{-1} \circ \Phi \circ \Psi\right)$. The previous result applies to $T_{1}$ to prove that $T_{1}$ is nilpotent. It follows that $T$ is nilpotent. This completes the proof.

REMARK. If $T$ is an automorphism satisfying case (iv), then $T \circ T$ falls into case (iii). Let $\Phi$ be induced by $T$. If $\Phi \circ \Phi$ has a fixed point in $\Delta$ or $\Delta_{p}$, then Theorem 2 applies and shows that $T^{2 n}=I$ for some integer $n>0$.

The next example shows that for particular $A(p)$ there exists automorphisms which are not nilpotent.

EXAMPLE 4. This construction employs the methods of [9], Lemma 5. Let $\alpha$ be a conformal map of $\Delta$ which has two fixed 
points on $\Gamma$. Let $L(z)$ be a linear fractional map taking $\Delta$ to the upper half plane and taking the two fixed points of $\alpha$ to 0 and $\infty$. Then $g=L \circ \alpha \circ L^{-1}$ has fixed points 0 and $\infty$ and $\operatorname{Im}[g(i)]>0$. Hence, $g(z)=a z$ with $a>0$ and $a \neq 1$.

We will construct a strictly increasing, continuous, singular function $h(x)$ on $\boldsymbol{R}$ such that $h \circ g=g \circ h$. We assume $a>1$, the case $a<1$ being similar. Let $h_{0}(x)$ be a strictly increasing, continuous, singular function mapping $[1, a]$ onto itself. Define $h(x)$ on $(0, \infty)$ by setting

$$
h(x)=a^{n} h_{0}\left(a^{-n} x\right) \text { for } x \in\left[a^{n}, a^{n+1}\right]
$$

where $n$ takes integer values. Extended $h(x)$ to $\boldsymbol{R}$ by setting $h(0)=0$ and $h(x)=-h(-x)$ if $x<0$. It can be checked that $h(x)$ satisfies the specified relationship with $g(x)$.

We pull back to $\Gamma$ by defining $p\left(e^{i t}\right)=L^{-1} \circ h \circ L\left(e^{i t}\right)$. Then $p\left(e^{i t}\right)$ is singular and $p \circ \alpha\left(e^{i t}\right)=\alpha \circ p\left(e^{i t}\right)$. It follows that the map $T$ on $A(p)$ defined by $T(f)=f \circ \alpha$ is an automorphism of $A(p)$.

Let $g_{n}$ be the composition of $g$ with itself $n$ times. We refer to such a composition as the $n$th iterate of $g$. Since $g_{n}(z)$ converges to 0 or $\infty$ for each $z$, the iterates $\alpha_{n}$ of $\alpha$ converge pointwise on $\Gamma$ to the fixed points of $\alpha$. In particular, $\alpha_{n}(z)$ is not the identity function for any $n$. Hence, $T^{n} \neq I$ for every $n$. This completes Example 4.

We conclude this section by suggesting two problems. Let Aut $(A(p))$ refer to the group of automorphisms of $A(p)$.

Problem 1. Is it possible to construct a $p\left(e^{i t}\right)$ so that $\operatorname{Aut}(A(p))$ consists of only the identity?

Problem 2. Let the sets $N_{\varepsilon, f}(T)=\{U \in \operatorname{Aut}(A(p)): \| T(f)-$ $U(f) \|<\varepsilon\}$ for $\varepsilon>0, f \in A(p)$, and $T \in \operatorname{Aut}(A(p))$ be a subbase for a topology on $\operatorname{Aut}(A(p))$. With this topology $\operatorname{Aut}(A(p))$ is a topological group. Is Aut $(A(p))$ discrete?

4. Spectra of endomorphisms of $A(p)$. We apply the methods of Kamowitz [4], [5] to obtain information about the spectra of endomorphisms of $A(p)$. Let $T$ be an endomorphism. The spectrum of $T$, denoted by $\sigma(T)$, is given by $\{\lambda \in C: \lambda-T$ is not invertible $\}$. Equivalently, $\lambda \in \sigma(T)$ if $\lambda-T$ is not 1-to-1 or not onto.

We consider the situation where an endomorphism has an induced map which has a fixed point in $\Delta$. Thus, we consider a subset of the endomorphisms satisfying case (i) or (iii). The next lemma shows that we can assume the origin is the point fixed by the induced map. (Cf. [4], Lemma 1.) 
LEMMA 1. Let $T$ be an endomorphism of $A(p)$ with induced map $\Phi$ where $\Phi\left(z_{0}\right)=z_{0} \in \Delta$. Let $g(z)$ be a conformal map of $\Delta$ taking 0 to $z_{0}$ and set $q=g^{-1} \circ p \circ g$. If $T_{1}$ is the endomorphism of $A(q)$ defined by $T_{1}(f)=f\left(g^{-1} \circ \Phi \circ g\right)$, then the induced map $\Phi_{1}$ satisfies $\Phi_{1}(0)=0, \Phi_{1}^{\prime}(0)=\Phi^{\prime}\left(z_{0}\right)$, and furthermore, $\sigma(T)=\sigma\left(T_{1}\right)$.

We omit the proof of Lemma 1 . The approach developed by Kamowitz in [4] for describing the spectra of endomorphisms of $A(\Delta)$ can now be adapted with minor modifications to the endomorphisms of $A(p)$.

LemMa 2. Let $T$ be an endomorphism of $A(p)$ with induced map $\Phi$ where $\Phi(0)=0$. Then $\sigma(T) \supset\left\{\left(\Phi^{\prime}(0)\right)^{n}: n\right.$ is a positive integer $\}$.

Proof. We have $\Phi(\Delta) \subset \Delta$ since $\Phi$ fixes the origin. The case where $\Phi^{\prime}(0)=0$ is obvious. Consider $0<\left|\Phi^{\prime}(0)\right| \leqq 1$. Given an integer $k>0$ let $g(z)=z^{k} h(z) \in A(p)$ where $h(0) \neq 0$. Then following [4], Lemma 2 we may show that $\left(\Phi^{\prime}(0)\right)^{k} f-f \circ \Phi \neq g$ for any $f \in A(p)$. Hence, $\left(\Phi^{\prime}(0)\right)^{k}-T$ is not onto and $\left(\Phi^{\prime}(0)\right)^{k} \in \sigma(T)$.

In view of Theorem 2 and the spectral mapping theorem we conclude:

Corollary. Let $T$ be an automorphism of $A(p)$ with induced map $\Phi$ where $\Phi(0)=0$. Let $c=\Phi^{\prime}(0)$. Then there is an integer $n>0$ such that $c^{n}=1$ and $\sigma(T)=\left\{1, c, c^{2}, \cdots, c^{n-1}\right\}$.

Let $T$ be an endomorphism of $A(p)$ with induced map $\Phi$ with $\Phi(\Delta) \subset \Delta$. Let $\Phi_{n}$ denote the $n$th iterate of $\Phi$. Then $\bigcap_{n=1}^{\infty} \Phi_{n}(\bar{\Delta})$ is called the fixed set of $\Phi$. The fixed set is compact and connected.

THEOREM 3. Let $T$ be an endomorphism of $A(p)$ with induced map $\Phi$ where $\Phi\left(z_{0}\right)=z_{0} \in \Delta$ and suppose the fixed set is infinite. If $T$ is not an automorphism, then $\sigma(T)=\bar{\Delta}$.

Proof. Apply the method of [4], Theorem 7 after replacing $g(z)=z^{\nu}$ by $g(z)=z^{\nu} h(z) \in A(p)$ where $\|h\| \leqq 1$.

Lemma 3. Let $T$ be an endomorphism of $A(p)$ with induced map $\Phi$ where $\Phi(\Delta) \subset \Delta$ and $\Phi\left(\Delta_{p}\right) \subset \Delta$. Suppose $\Phi(0)=0$. Let $\nu$ be a positive integer and suppose every $f \in A(p)$ with a zero of order at least $(\nu+1)$ at 0 is in the range of $\lambda-T$ where $\lambda \notin\left\{\left(\Phi^{\prime}(0)\right)^{n}: n\right.$ is a positive integer $\} \cup\{0,1\}$. Then $\lambda-T$ is onto.

Proof. Let $z^{\nu} q(z) \in A(p)$ where $q(0)=1$. Define $g(z)=\Phi(z)^{\nu}-$ 
$\Phi^{\prime}(0)^{\nu} z^{\nu} q(z)$. By assumption $T$ falls into case (i) and so $\Phi \in A(p)$. Thus, $g \in A(p)$. Also, $g$ vanishes at $z=0$ to order at least $(\nu+1)$. By hypothesis there is $h \in A(p)$ such that $(\lambda-T) h=g$. Set $f=$ $h+z^{\nu} q$. Then

$$
\begin{aligned}
& (\lambda-T) f=(\lambda-T) h+(\lambda-T) z^{\nu} q \\
& \quad=\Phi(z)^{\nu}-\left(\Phi^{\prime}(0)\right)^{\nu} z^{\nu} q(z)+\lambda z^{\nu} q(z)-\Phi(z)^{\nu} q(\Phi(z)) \\
& \quad=\Phi(z)^{\nu}(1-q(\Phi(z)))+\left(\lambda-\Phi^{\prime}(0)^{\nu}\right) z^{\nu} q(z) .
\end{aligned}
$$

Since $(\lambda-T) f$ and $z^{\nu} q(z)$ belong to $A(p)$, so does $\Phi(z)^{\nu}(1-q(\Phi(z)))$. Also, $\Phi(z)^{\nu}(1-q(\Phi(z)))$ vanishes to order at least $(v+1)$ at the origin and so $\Phi(z)^{\nu}(1-q(\Phi(z)))$ belongs to the range of $\lambda-T$. Since $\lambda-\Phi^{\prime}(0)^{\nu} \neq 0$, we conclude $z^{\nu} q(z)$ belongs the range of $\lambda-T$.

Let $f \in A(p)$ vanish to order at least $\nu$ at the origin. We will show that $f$ belongs to the range of $\lambda-T$. We have $f(z)=a z^{\nu}+$ $r(z)$ and $a z^{\nu} q(z)=a z^{\nu}+s(z)$ where both $r(z)$ and $s(z)$ vanish to order at least $(\nu+1)$ at the origin. Then $f(z)=a z^{\nu}+s(z)-s(z)+r(z)=$ $a z^{\nu} q(z)-(s(z)-r(z))$. Since $f(z)$ and $a z^{\nu} q(z)$ belong to $A(p)$, so does $s(z)-r(z)$. But then $s(z)-r(z)$ belongs to the range of $\lambda-T$. Also, $a z^{\nu} q(z)$ belongs to the range of $\lambda-T$. Hence, $f(z)$ belongs to the range of $\lambda-T$. Now by use of induction we see that $\lambda-T$ is onto. This completes the proof.

THEOREM 4. Let $T$ be an endomorphism of $A(p)$ with induced map $\Phi$ where $\Phi\left(z_{0}\right)=z_{0} \in \Delta$. Suppose the fixed set of $\Phi$ is $\left\{z_{0}\right\}$. Then $\sigma(T)=\left\{\left(\Phi^{\prime}\left(z_{0}\right)\right)^{n}: n\right.$ is a positive integer $\} \cup\{0,1\}$.

Proof. Since the fixed set is $\left\{z_{0}\right\}, \Phi$ must satisfy case (i) for under case (iii) $\Phi$ maps $\bar{\Delta}$ onto itself. In particular, Lemma 3 applies. The method of [4], Theorem 9 can now be applied with minor modifications.

REMARK. The spectra of endomorphisms whose induced map fixes a point of $\Delta_{p}$ can be described in a manner entirely parallel to the foregoing work. If $T$ is an endomorphism of $A(p)$ and if the induced map $\Phi$ fixes a point of $\Delta_{p}$, then Theorems 3 and 4 can be restated with $\Theta=\Psi^{-1} \circ \Phi \circ \Psi$ replacing $\Phi$ and with $\bigcap_{n=1}^{\infty} \Theta_{n}(\bar{A})$ taking the role of the fixed set.

We give one result describing the spectra of automorphisms satisfying case (iv).

THEOREM 5. Let $T$ be an automorphism of $A(p)$ with induced map $\Phi$ where $\Phi(\Delta) \subset \Delta_{p}$ and $\Phi\left(\Delta_{p}\right) \subset \Delta$. Suppose $\Phi \circ \Phi\left(z_{0}\right)=z_{0} \in \Delta$. If $T^{2} \neq I$, then $\sigma(T)=\left\{\lambda: \lambda^{2} \in \sigma\left(T^{2}\right)\right\}$. 
Proof. Using Lemma 1 and the corollary to Lemma 2 we conclude that there is an integer $n>1$ where $c^{n}=1$ and $\sigma\left(T^{2}\right)=$ $\left\{1, c, c^{2}, \cdots, c^{n-1}\right\}$ for $c=(\Phi \circ \Phi)^{\prime}\left(z_{0}\right)$. By the spectral mapping theorem $\sigma\left(T^{2}\right)=(\sigma(T))^{2}$. So, either $\sqrt{c}$ or $-\sqrt{c}$ belongs to $\sigma(T)$. According to [6], Theorem 1, $\sigma(T)$ is a finite subgroups of $\Gamma$. Since both $\sqrt{c}$ and $-\sqrt{c}$ generate the $2 n$th roots of unity, we see that the $2 n$th roots of unity comprise $\sigma(T)$. This completes the proof.

EXAMPLE 5. We construct a singular homeomorphism $p\left(e^{i t}\right)$ of $I$ such that $p \circ p\left(e^{i t}\right)=-e^{i t}$. Let $h_{1}(x):[0, \pi / 2] \rightarrow[\pi / 2, \pi]$ be strictly increasing, continuous and singular. Then for integer values of $j$ define $h_{j}(x):[\pi(j-1) / 2, \pi j / 2] \rightarrow[\pi j / 2, \pi(j+1) / 2]$ by

$$
h_{j}(x)=h_{j-1}^{-1}(x)+\pi \quad \text { for } j \geqq 2
$$

and

$$
h_{j}(x)=h_{j+1}^{-1}(x+\pi) \text { for } j \leqq 0 .
$$

Now use the $h_{j}(x)$ to define $h(x): \boldsymbol{R} \rightarrow \boldsymbol{R}$. It may be shown that $h \circ h(x)=x+\pi$. Set $p\left(e^{i t}\right)=\exp (i h(t))$ to obtain the required map.

The map $p\left(e^{i t}\right)$ gives rise to an algebra $A(p)$ and automorphism $T(f)=f \circ p$. The induced map $\Phi$ satisfies case (iv) and $\Phi \circ \Phi(z)=-z$. Then $\sigma\left(T^{2}\right)=\{1,-1\}$ and by Theorem $5 \sigma(T)=\{1, i,-1,-i\}$.

REMARK. It can be verified that the spectrum of the automorphism in Example 3 is given by $\{1,-1\}$.

\section{REFERENCES}

1. R. G. Blumenthal, Maximality in function algebras, Canad. J. Math., 22 (1970), 1002-1004.

2. - The closed ideals of some Dirichlet and hypo-Dirichlet algebras, Proc. Amer. Math. Soc., 82 (1972), 469-471.

3. A. Browder and J. Wermer, A method of constructing Dirichlet algebras, Proc. Amer. Math. Soc., 15 (1964), 546-552.

4. H. Kamowitz, The spectra of endomorphisms of the disc algebra, Pacific J. Math., 46 (1973), 433-440.

5. - The spectra of endomorphisms of algebras of analytic functions, Pacific J. Math., 66 (1976), 433-442.

6. H. Kamowitz and S. Scheinberg, The spectrum of automorphisms of Banach algebras, J. Functional Analysis, 4 (1969), 268-276.

7. G. M. Leibowitz, Lectures on Complex Function Algebras, Scott, Foresman and Company, Glenview, Ill., 1970.

8. B. Lund, Functions belonging to a Dirichlet subalgebra of the disk algebra, Canad. Math. Bull., 18 (1975), 375-377.

9. A. Shields, On fixed points of commuting analytic functions, Proc. Amer. Math. Soc., 15 (1964), 703-706. 
10. L. Takacs, An increasing continuous singular function, Amer. Math. Monthly 85 (1978), 35-36.

Received December 5, 1978.

23595 Stonehenge Buvd

Novi, MI 48050 
.././. ./FrontMatter/paper .pdf 


\section{Pacific Journal of Mathematics \\ Vol. 87, No. $2 \quad$ February, 1980}

Theagenis Abatzoglou, Unique best approximation from a $C^{2}$-manifold in Hilbert space ................................. 233

Gerald Arthur Anderson, $\Lambda$-homology cobordism bundles............. 245

Eric Bedford, Holomorphic mapping of products of annuli in $\mathbf{C}^{n} \ldots \ldots \ldots 271$

Gunnar Carlsson, On the stable splitting of $b o \wedge b o$ and torsion operations in connective $K$-theory .......................... 283

Lester Eli Dubins and David Samuel McIntyre Margolies, Naturally integrable functions ................................. 299

Leo Egghe, The Radon-Nikodým property, $\sigma$-dentability and martingales in locally convex spaces ............................. 313

Irving Leonard Glicksberg, Maps preserving translates of a function ..... 323

Hugh M. Hilden and Robert D. Little, Cobordism of branched covering

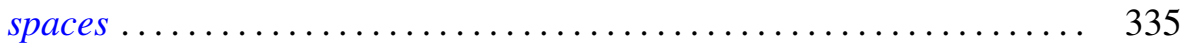

Russell Allan Johnson, Almost-periodic functions with unbounded integral .......................................... 347

Bruce Stephen Lund, The endomorphisms of a Dirichlet algebra ........ 363

John Henry McCleary, Mod $p$ decompositions of H-spaces; another approach........................................ 373

Arlan Bruce Ramsay, Subobjects of virtual groups ................ 389

Thomas R. Savage, Generalized inverses in regular rings ............ 455 Jaak Vilms, On curvature operators of bounded rank ......... 